\title{
Determination of carrier lifetime and mobility in colloidal quantum dot films via impedance spectroscopy
}

\author{
Arup K. Rath, ${ }^{\text {a) }}$ Tania Lasanta, Maria Bernechea, Silke L. Diedenhofen, \\ and Gerasimos Konstantatos ${ }^{\text {b) }}$ \\ ICFO-Institut de Ciencies Fotoniques, Mediterranean Technology Park, Castelldefels, \\ Barcelona 08860, Spain
}

(Received 2 December 2013; accepted 21 January 2014; published online 11 February 2014)

\begin{abstract}
Impedance Spectroscopy (IS) proves to be a powerful tool for the determination of carrier lifetime and majority carrier mobility in colloidal quantum dot films. We employ IS to determine the carrier lifetime in $\mathrm{PbS}$ quantum dot Schottky solar cells with $\mathrm{Al}$ and we verify the validity of the technique via transient photovoltage. We also present a simple approach based on an RC model that allows the determination of carrier mobility in $\mathrm{PbS}$ quantum dot films and we corroborate the results via comparison with space charge limited measurements. In summary, we demonstrate the potential of IS to characterize key-to-photovoltaics optoelectronic properties, carrier lifetime, and mobility, in a facile way. (C) 2014 AIP Publishing LLC. [http://dx.doi.org/10.1063/1.4865089]
\end{abstract}

Colloidal quantum dot optoelectronics ${ }^{1}$ and photovoltaics $^{2-6}$ have seen tremendous progress over the last decade. Behind this progress lies the better understanding of the optoelectronic properties of these materials, particularly those immediately relevant to optoelectronic devices, i.e., carrier lifetime and carrier mobility. A plethora of techniques have been deployed to measure these parameters including transient photovoltage ${ }^{6-8}$ for carrier lifetime and time-offlight, ${ }^{3}$ field-effect-transistor (FET) ${ }^{9}$ and space charge limited current (SCLC) ${ }^{10}$ methods for carrier mobility estimation. Ideally, these parameters should be measurable in structures that are similar to the actual devices and most importantly measured at experimental conditions similar to the operating conditions of the device. Impedance Spectroscopy (IS) has been employed to measure these parameters in thin film devices, particularly thin film solar cells for over a decade. ${ }^{11-14}$ It is considered a powerful technique for it yields a number of electronic parameters from a single set of experiments ${ }^{15}$ which would otherwise require multiple experimental techniques to be realized. ${ }^{7}$ Most importantly, this technique can be used directly on the solar cell devices without any significant change in device architecture and allows the measurement of these parameters under different experimental conditions. ${ }^{11,15}$

Despite the significant advantages, this technique has been underused in characterizing quantum dot thin film solar cells due to the complexity involved in the interpretation of experimental results. ${ }^{15-17}$ The power conversion efficiency of a solar cell is largely dependent on the product of carrier mobility and carrier lifetime of the active semiconductor layer. ${ }^{18}$ It is intriguing therefore to be able to determine carrier lifetime and mobility of the active material in a facile way. In this study, we have determined physical parameters like carrier lifetime, transient time, mobility, and diffusion length solely based on IS measurements. For this study, we have chosen the Schottky diode structure formed by $\mathrm{p}$-type $\mathrm{PbS}$

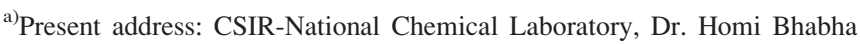
Road, Pune 411008, India

b) gerasimos.konstantatos@icfo.es
}

QDs and an LiF/Al metal contact, for it is the simplest possible device configuration for a QD solar cell and is suitable for the present study to establish our proposed hypothesis. ${ }^{19}$

We build our study on the model proposed by MoraSeró et al. ${ }^{12}$ Based on this, the carrier lifetime can be determined as the product of recombination resistance $\left(R_{\text {rec }}\right)$ and chemical capacitance $\left(C_{\mu}\right)$. The equivalent circuit diagram of the device is shown in Figure 1. Carrier lifetime is determined at open circuit condition, since, at this condition, no current flows through the external circuit and all the photogenerated carries recombine through the recombination resistance $R_{\text {rec }}$. To ensure that no current is flowing through the external circuit, we apply external bias equal to the open circuit voltage to our device under illumination and measure the frequency dependent impedance of the device.

Figure 2(a) shows the imaginary impedance versus real impedance plot for different illumination intensities at open

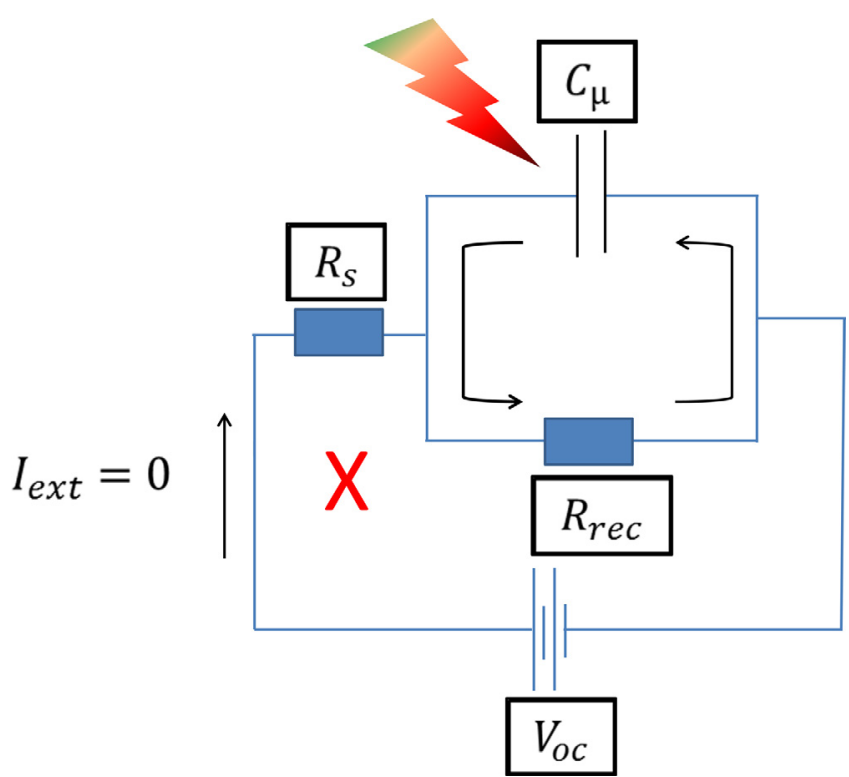

FIG. 1. Equivalent circuit diagram of a solar cell with external bias that consists of the series resistance $R_{s}$, Recombination resistance $R_{\text {rec }}$, and the chemical capacitance $C_{\mu}$. 
circuit condition. $R_{\text {rec }}$ is calculated from the difference of the real impedance values measured at frequency of $20 \mathrm{~Hz}$ and $2 \mathrm{MHz}$. Figure 2(b) illustrates the dependence of $R_{\text {rec }}$ on illumination intensity. Recombination resistance decreases with increasing intensity due to the enhancement in recombination current from increasing population of photocarriers at the quasi-Fermi levels. The capacitance measured at different illumination intensities at open circuit condition is shown in Figure 2(c). Therein, three distinct regions as indicated by lines can be identified: (1) In the low frequency region, the capacitance decreases with increasing frequency. In this region, the capacitance depends also on light intensity, increasing at higher intensities. (2) In the intermediate frequency region, the capacitance is constant and it is independent of both frequency and intensity of the incident light. (3) In the high frequency region, capacitance decreases with increasing frequency, yet it is independent of light intensity. Figure 2(d) shows the carrier lifetime calculated as the product of $C_{\mu}$ and $R_{r e c}$ vs frequency. The product is not constant in the high and low frequency regimes, whereas it is constant in the intermediate frequency regime. Here, we posit that the $C_{\mu} \times R_{\text {rec }}$ product yields the carrier lifetime in the intermediate frequency regime, based on the proposed model shown in Fig. 2(e). ${ }^{20}$ Fig. 2(e) shows the band diagram of our Schottky device at equilibrium. $E_{c}, E_{v}$, and $E_{f}$ denote the conduction band edge, valence band edge, and Fermi level, respectively. Nanocrystalline and quantum dot solids are known to possess midgap trap states within their (a)

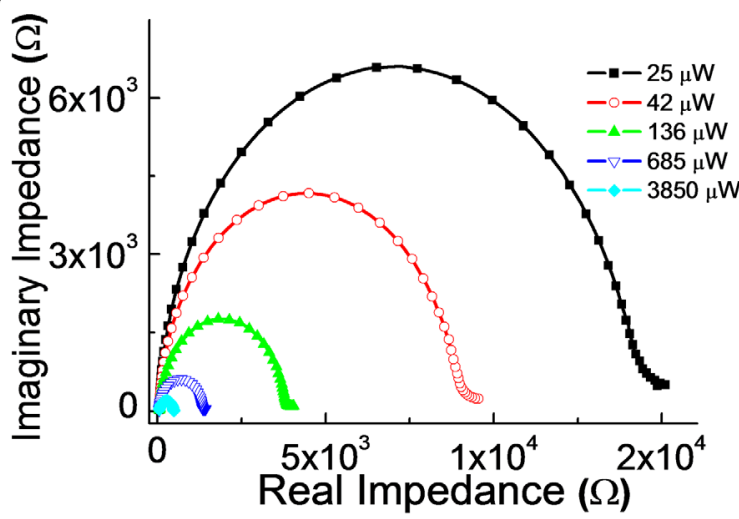

(c)

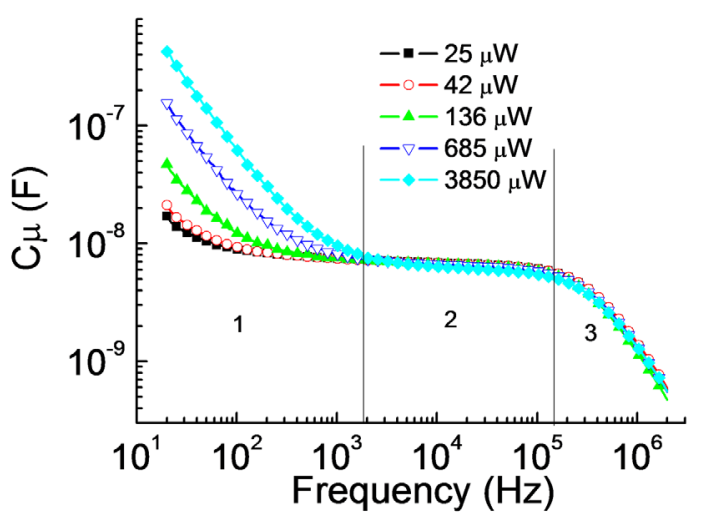

(e)

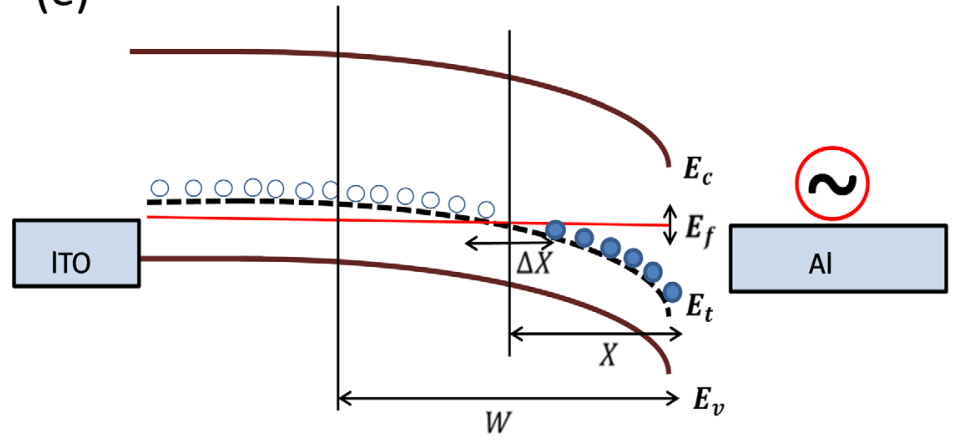

(b)

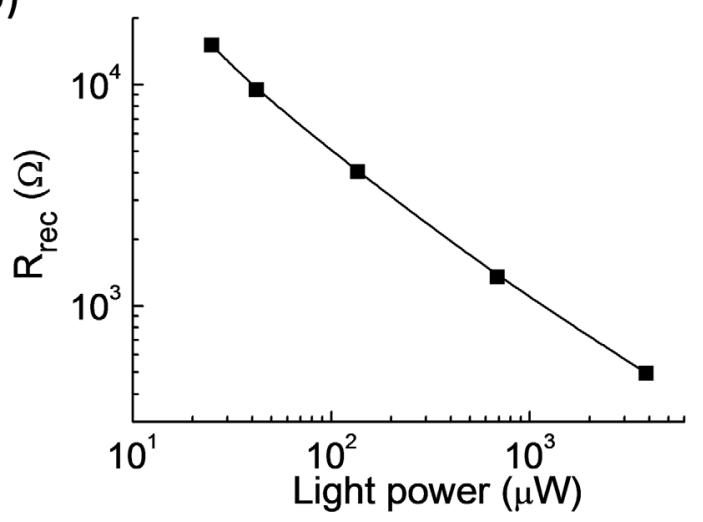

(d)

(f)
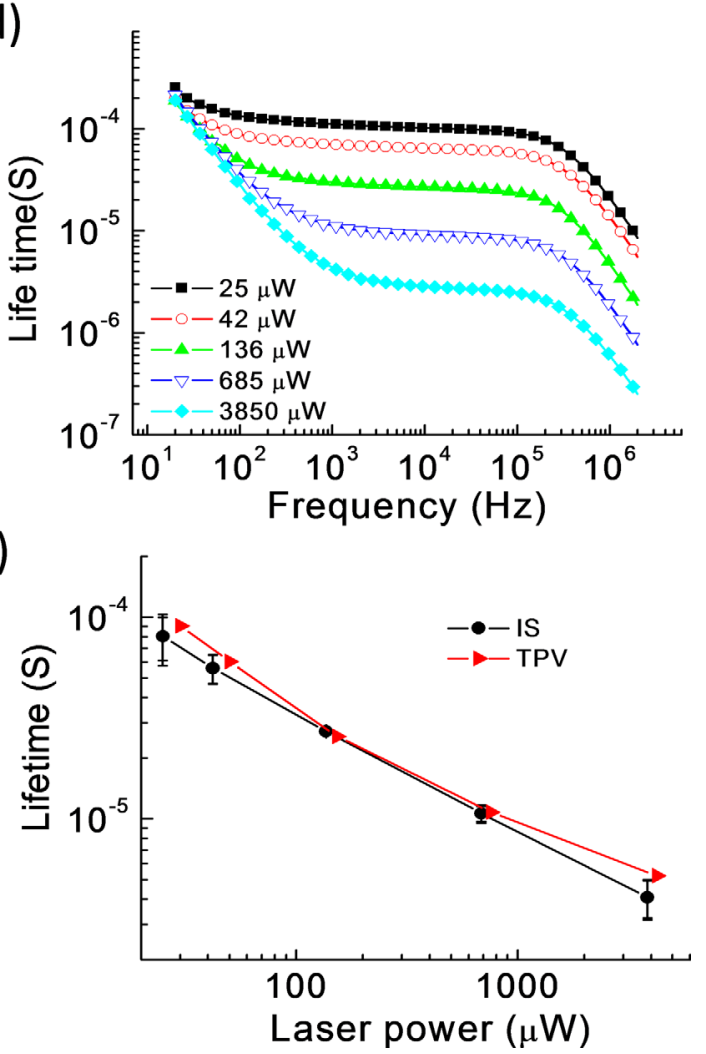

FIG. 2. (a) Plot of real impedance Vs Imaginary impedance taken at open circuit condition under illumination. Colours denote different light intensities. (b) $R_{\text {rec }}$ Vs light intensity variation in $\log -\log$ scale. (c) Capacitance vs frequency graph at different light intensities under open circuit condition. Three regions are identified by two black lines and marked as 1, 2, 3 to guide the eye. (d) Carrier lifetime vs frequency plot- the different colors indicate different light intensities. (e) Band alignment and trap distribution under the action of applied voltage. (f) Comparison of lifetime calculated from IS and transient photo voltage (TPV). 
bandgap due to surface dangling bonds. Herein, we consider a single trap state level, to demonstrate the rationale, denoted by $E_{t} . \mathrm{W}$ is the depletion width and $\mathrm{X}$ denotes the thickness within the depletion region in which the electron trap states are filled by electron population by the $\mathrm{Al}$ in the formation of the Schottky junction. Thus, trap states that lie within the thickness $\mathrm{X}$ are filled whereas beyond this point are empty. On the application of an AC signal, Fermi energy position of the semiconductor layer $E_{f}$ will vary, and, as a result, the number of populated trap states, below the Fermi level, will be modulated accordingly. A larger number of charges under the Fermi level reduces the depletion width and raises the capacitance. ${ }^{20-22}$ These deep midgap states are highly localized and their contribution to charge collection is insignificant, thus their contribution in the determination of the effective carrier lifetime can be neglected. In the intermediate frequency regime, contribution of deep-trapped carriers to capacitance decreases due to the finite time constant associated with the charging and discharging of these deep level states. Above a certain frequency, deep trapped carriers cannot follow the applied signal, and the capacitance of the device should remain constant with respect to frequency. Capacitance in this regime should only depend on the free carrier density and can be given as ${ }^{20} C_{\mu}=\varepsilon_{\mathrm{o}} \varepsilon_{s} A / W$, where $A$ is the geometric area, $W$ the depletion width, and $\varepsilon_{s}$ the dielectric constant of the film. Hence, we posit that the effective carrier lifetime can be determined in this intermediate frequency region. In the high frequency regime $\left(>10^{5} \mathrm{~Hz}\right)$, the capacitance becomes again frequency dependent as the applied frequency becomes comparable to the transit time of (a)
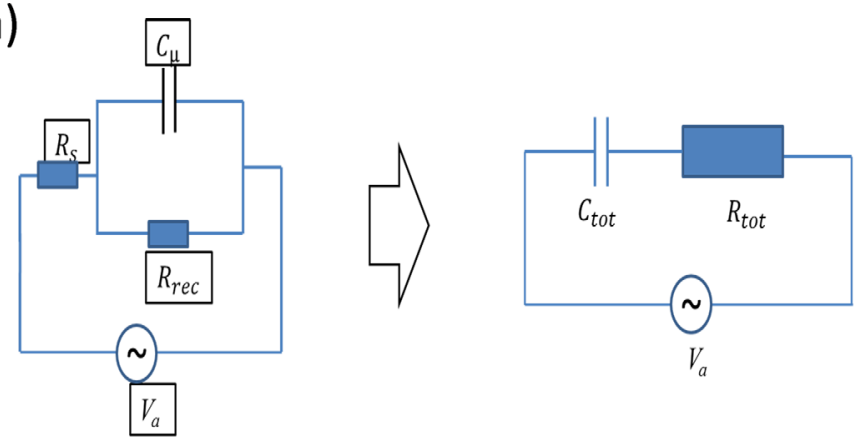

(c)

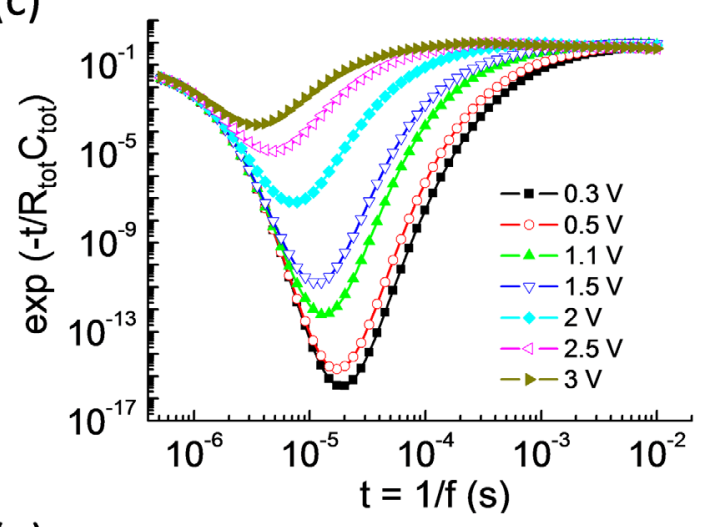

(e)

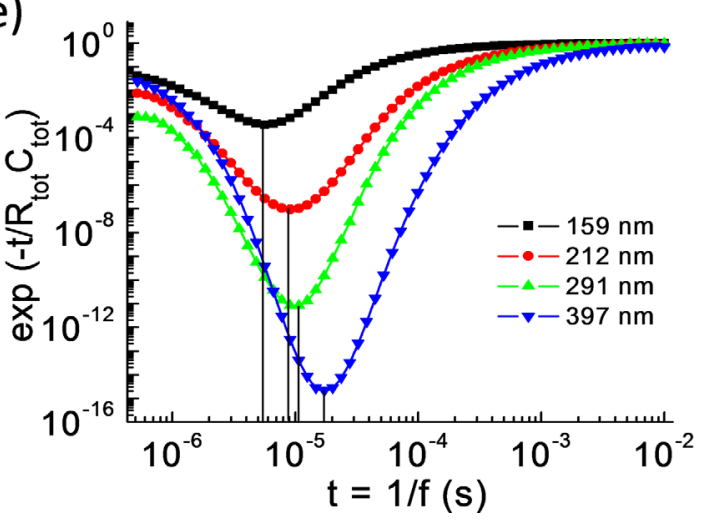

(b)

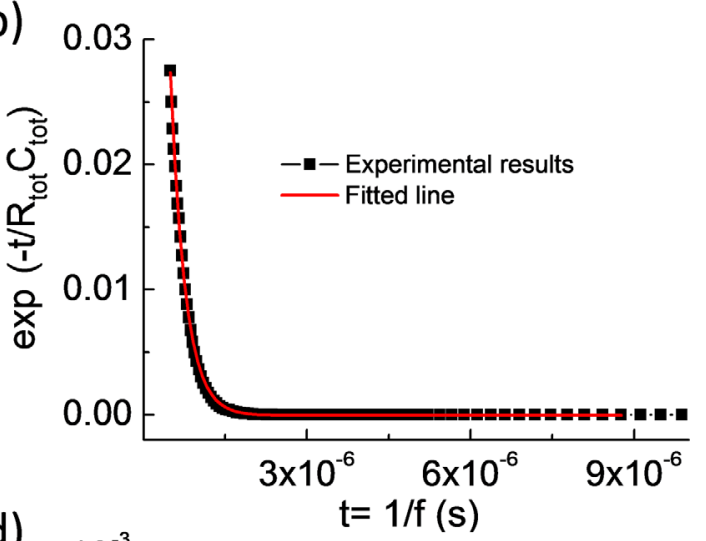

(d)

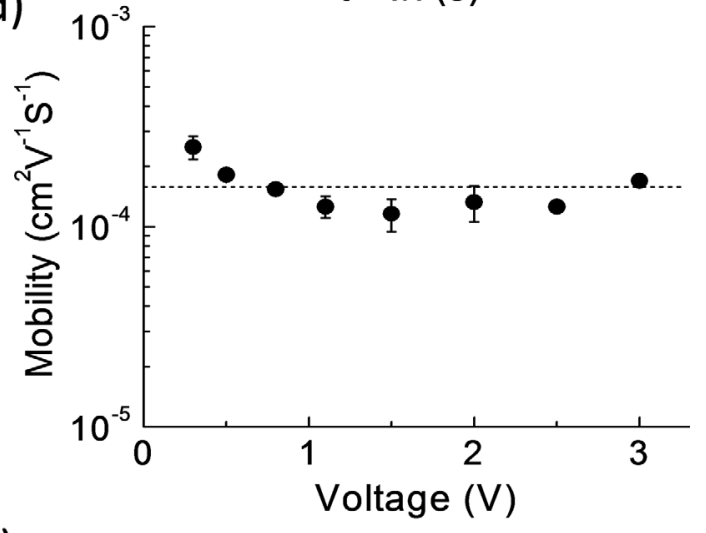

(f)

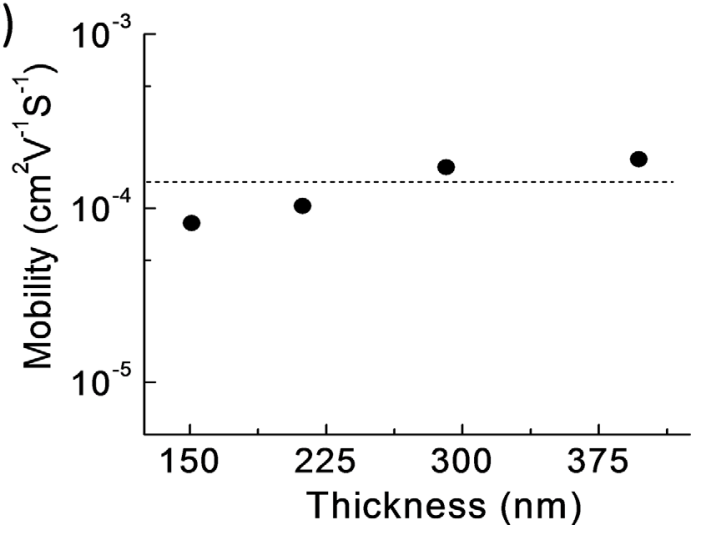

FIG. 3. (a) The equivalent circuit of a solar cell and the equivalent circuit represented as a series combination of a resistance and a capacitance. (b) The exponential decay of $\mathrm{e}^{-\frac{\mathrm{t}}{\mathrm{R}_{\mathrm{tot}} \times \mathrm{C}_{\mathrm{tot}}}}$ Vs time at short time scales. Black squares represent the experimental data points and red line represents the fitted line for single exponential decay. (c) $\mathrm{e}^{-\frac{\mathrm{t}}{\mathrm{R}_{\mathrm{tot}} \times \mathrm{C}_{\mathrm{tot}}}}$ versus time for the entire time scale (i.e., frequency range of $20 \mathrm{~Hz}-2 \mathrm{MHz}$ ) taken at different applied biases for $397 \mathrm{~nm}$ thick device. (d) The invariance of mobility at different applied bias. (e) $\mathrm{e}^{-\frac{1}{\mathrm{R}_{\mathrm{tot}} \times \mathrm{C}_{\mathrm{tot}}}}$ versus time plot for different thicknesses taken at $0.5 \mathrm{~V}$ applied bias. (f) Mobility vs thickness plot showing no significant change in mobility measured for varying thickness of $\mathrm{PbS}$ layer. 
the free carriers inside the device. From Fig. 2(d), the carrier lifetime is determined at the frequency regime where lifetime is constant with respect to frequency. The carrier lifetime for different illumination intensities has been plotted in Fig. 2(f) along with the carrier lifetime measured via transient photovoltage measurements ${ }^{6,7}$ and are found to be in good agreement. To further corroborate our studies and to study the thickness dependence variability, we characterized devices with varying thickness. The I-V and performance characteristics of the devices ${ }^{19}$ compare nicely with prior reports of these structures. ${ }^{23}$ No significant variation of the carrier lifetime with device thickness was observed. ${ }^{19}$

We turn now to the determination of another significant parameter that IS can provide us with: Majority carrier mobility. Several attempts have been made to determine carrier mobility by determining carrier transit time from impedance spectroscopy measurements. ${ }^{17,24,25}$ Prior reports on the determination of carrier mobility in organic semiconductors from IS have relied on the correlation of the frequency at which negative differential susceptance is observed and the transit time measured via time-of-flight techniques. ${ }^{17,24} \mathrm{In}$ the current report, we propose an alternative model in which the transit time of majority carriers is determined directly from IS measurements. To do so, we have built devices that consist of ITO-PbS QDs-Au to provide Ohmic contacts on both sides of the PbS layer. ${ }^{19,24}$ The equivalent circuit model of the device is shown in Figure 3(a). The total impedance can be written as

$$
\mathrm{Z}=\mathrm{R}_{\mathrm{s}}+\frac{1}{\frac{1}{\mathrm{R}_{\mathrm{sh}}}+\mathrm{j} \omega \mathrm{C}_{\mu}}=\mathrm{R}_{\mathrm{tot}}+\frac{1}{\mathrm{j} \omega \mathrm{C}_{\mathrm{tot}}},
$$

where $R_{t o t}=R_{s}+\frac{R_{s h}}{1+\omega^{2} C_{\mu}^{2} R_{s h}^{2}}$ is the real part of the total impedance and $C_{\text {tot }}=\frac{1+\omega^{2} C_{\mu}^{2} R_{s h}^{2}}{\omega^{2} C_{\mu} R_{s h}^{2}}$ is the total capacitance of the device. Where $R_{s}$ is the series resistance and $R_{s h}$ is the shunt resistance of the cell.

Equation (1) indicates that the equivalent circuit can be redrawn as a series combination of $R_{t o t}$ and $C_{t o t}$. The series equivalent circuit based on Eq. (1) is shown in Figure 3(a). According to this, the voltage across the capacitor at a certain time can be written as

$$
V_{c}=V_{b}\left(1-e^{-\frac{t}{R_{t o t} \times C_{t o t}}}\right),
$$

where $\mathrm{V}_{\mathrm{b}}$ is the applied bias. Both $R_{t o t}$ and $C_{t o t}$ are dependent on time $t$, as $t=\frac{1}{f}$, where $f$ is the applied frequency. We will now refer to this study in the time domain for convenience. At timescales shorter than the transit time of the carriers in the device (thus at high modulation frequencies), the injected carriers from one contact cannot reach the opposite electrode. Therefore net current does not flow through the device, and the PbS QD layer behaves like a dielectric layer. The voltage then builds across the PbS layer according to Eq. (2), and the term $e^{-\frac{t}{R_{\text {tot }} \times C_{\text {tot }}}}$ follows a simple exponential decay with time, as shown in Fig. 3(b). At progressively longer timescales (i.e., progressively lower frequencies), there is a point at which the modulation frequency is such that the carriers can follow the applied electric field, i.e., enough time is provided to them to reach the electrodes, which will be equivalent to the transit time in the device. Passed this point carriers will no longer built up across the $\mathrm{PbS}$ layer, and therefore the term $e^{-\frac{t}{R_{\text {tot }} \times C_{\text {tot }}}}$ will recover. The frequency at which this transition occurs $f_{\text {transit }}=1 / t_{\text {transit }}$ determines the transit time, and can thus yield the mobility of the carriers as $t_{\text {transit }}=\frac{d}{\mu E}=\frac{d^{2}}{\mu V}$ Fig. 3(c) shows the dependence of this transition time on the applied electric field based on which the transit time scales linearly with the applied bias. As expected, then, the mobility is invariant with the applied bias, as shown in Fig. 3(d). To further corroborate the model, we also studied the dependence of the transit time on the thickness of the device at a fixed bias. As shown in Fig. 3(e), the transit time varies linearly with the device thickness leading to a constant mobility as a function of thickness (Fig. 3(f)). The resultant hole mobility value for ethane-dithiol (EDT) treated PbS QD films is found to be $1.37 \times 10^{-4} \mathrm{~cm}^{2} / \mathrm{Vs}$, in good agreement with prior reports of this material. ${ }^{18}$ Taking the extracted lifetime and mobility together, we calculate the carrier diffusion length to be $\sim 60 \mathrm{~nm}$. Finally, we further confirmed the validity of our approach by measuring the mobility through the SCLC method. ${ }^{26}$ The average hole mobility is found to be 1.2 $\times 10^{-4} \mathrm{~cm}^{2} / \mathrm{Vs}$, in close agreement with our findings from the IS measurements. ${ }^{19}$

In summary, we have shown that IS can be a valuable tool for the characterization of colloidal quantum dot solar cells, for it can provide readily carrier lifetime and carrier mobility under experimental conditions similar to the ones under which solar cell devices operate. In this work, we measured carrier lifetime and majority carrier mobility in simple Schottky PbS quantum dot solar cells, paving the way for employing this technique for the characterization of more advanced colloidal quantum dot solar cell architectures. ${ }^{3-6}$

We acknowledge financial support from Fundació Privada Cellex Barcelona and partial funding from European Commission's Seventh Framework Programme for Research under Contract Nos. PIRG06-GA-2009-256355, PIEF-GA2011-298596, and FP7-ENERGY.2012.10.2.1 under Grant agreement No. 308997. This research was also supported in part by Strategic International Cooperative Program, Japan Science and Technology Agency (JST) and MICINN.

${ }^{1}$ G. Konstantatos and E. H. Sargent, Nat. Nanotechnol. 5(6), 391 (2010).

${ }^{2}$ J. Y. Kim, O. Voznyy, D. Zhitomirsky, and E. H. Sargent, Adv. Mater. 25(36), 4986 (2013).

${ }^{3}$ A. H. Ip, S. M. Thon, S. Hoogland, O. Voznyy, D. Zhitomirsky, R. Debnath, L. Levina, L. R. Rollny, G. H. Carey, A. Fischer, K. W. Kemp, I. J. Kramer, Z. Ning, A. J. Labelle, K. W. Chou, A. Amassian, and E. H. Sargent, Nat. Nanotechnol. 7(9), 577 (2012).

${ }^{4}$ O. E. Semonin, J. M. Luther, S. Choi, H. Y. Chen, J. B. Gao, A. J. Nozik, and M. C. Beard, Science 334(6062), 1530 (2011).

${ }^{5}$ J. M. Luther, M. Law, M. C. Beard, Q. Song, M. O. Reese, R. J. Ellingson, and A. J. Nozik, Nano Lett. 8(10), 3488 (2008).

${ }^{6}$ A. K. Rath, M. Bernechea, L. Martinez, F. P. Garcia de Arquer, J. Osmond, and G. Konstantatos, Nat. Photonics 6(8), 529 (2012).

${ }^{7}$ K. W. Johnston, A. G. Pattantyus-Abraham, J. P. Clifford, S. H. Myrskog, S. Hoogland, H. Shukla, E. J. D. Klem, L. Levina, and E. H. Sargent, Appl. Phys. Lett. 92(12), 122111 (2008).

${ }^{8}$ N. Zhao, T. P. Osedach, L. Y. Chang, S. M. Geyer, D. Wanger, M. T. Binda, A. C. Arango, M. G. Bawendi, and V. Bulovic, ACS Nano 4(7), 3743 (2010).

${ }^{9}$ D. V. Talapin and C. B. Murray, Science 310(5745), 86 (2005).

${ }^{10}$ B. S. Mashford, M. Stevenson, Z. Popovic, C. Hamilton, Z. Q. Zhou, C. Breen, J. Steckel, V. Bulovic, M. Bawendi, S. Coe-Sullivan, and P. T. Kazlas, Nat. Photonics 7(5), 407 (2013). 
${ }^{11}$ F. Fabregat-Santiago, G. Garcia-Belmonte, I. Mora-Sero, and J. Bisquert, Phys. Chem. Chem. Phys. 13(20), 9083 (2011).

${ }^{12}$ I. Mora-Sero, J. Bisquert, F. Fabregat-Santiago, G. Garcia-Belmonte, G. Zoppi, K. Durose, Y. Proskuryakov, I. Oja, A. Belaidi, T. Dittrich, R. Tena-Zaera, A. Katty, C. Levy-Clement, V. Barrioz, and S. J. C. Irvine, Nano Lett. 6(4), 640 (2006).

${ }^{13}$ T. Walter, R. Herberholz, C. Muller, and H. W. Schock, J. Appl. Phys. 80 (8), 4411 (1996).

${ }^{14}$ Y. Y. Proskuryakov, K. Durose, M. K. A. Turkestani, I. Mora-Sero, G. Garcia-Belmonte, F. Fabregat-Santiago, J. Bisquert, V. Barrioz, D. Lamb, S. J. C. Irvine, and E. W. Jones, J. Appl. Phys. 106(4), 044507 (2009).

${ }^{15}$ G. Garcia-Belmonte, P. P. Boix, J. Bisquert, M. Sessolo, and H. J. Bolink, Sol. Energy Mater. Sol. Cells 94(2), 366 (2010).

${ }^{16}$ W. L. Leong, S. R. Cowan, and A. J. Heeger, Adv. Energy Mater. 1(4), 517 (2011).

${ }^{17}$ N. D. Nguyen, M. Schmeits, and H. P. Loebl, Phys. Rev. B 75(7), 075307 (2007).
${ }^{18}$ K. S. Jeong, J, Tang, H, Liu, J, Kim, A. W. Schaefer, K. Kemp, L. Levina, X. Wang, S. Hoogland, R. Debnath, L. Brzozowski, E. H. Sargent, and J. B. Asbury, ACS Nano 6(1), 89 (2012).

${ }^{19}$ See supplementary material at http://dx.doi.org/10.1063/1.4865089 for device structure, I-V characteristics, carrier lifetime dependence on device thickness, and calculation of the mobility via the SCLC method.

${ }^{20}$ Y. Zohta, Solid-State Electron. 16(9), 1029 (1973).

${ }^{21}$ S. Khelifi, K. Decock, J. Lauwaert, H. Vrielinck, D. Spoltore, F. Piersimoni, J. Manca, A. Belghachi, and M. Burgelman, J. Appl. Phys. 110(9), 094509 (2011).

${ }^{22}$ G. Vincent, D. Bois, and P. Pinard, J. Appl. Phys. 46(12), 5173 (1975).

${ }^{23}$ J. Tang, X. Wang, L. Brzozowski, D. Aaron, R. Barkhouse, R. Debnath, L. Levina, and E. H. Sargent, Adv. Mater. 22(12), 1398 (2010).

${ }^{24}$ P. W. M. Blom, H. C. F. Martens, and J. N. Huiberts, Synth. Met. 121(1-3), 1621 (2001).

${ }^{25}$ S. W. Tsang, S. K. So, and J. B. Xu, J. Appl. Phys. 99(1), 013706 (2006).

${ }^{26}$ V. D. Mihailetchi, H. X. Xie, B. de Boer, L. J. A. Koster, and P. W. M. Blom, Adv. Funct. Mater. 16(5), 699 (2006). 